\title{
Tomografia Computadorizada das Artérias Coronárias: Novos Horizontes e Desafios
} Ver artigo relacionado
na página 327

\author{
Ibraim Pinto ${ }^{1}$
}

$\mathrm{A}$ tomografia computadorizada das artérias coronárias tem sido utilizada com crescente sucesso na prática clínica para a detecção nãoinvasiva da doença arterial coronária, em especial porque possibilita excluir com segurança a presença de obstruções no leito arterial coronário. Diversos trabalhos confirmaram que esse exame apresenta acurácia crescente e poder preditivo negativo superior a $90 \%{ }^{1,2}$. Contudo, à medida que a tecnologia evolui, e com o aumento da resolução temporal e espacial ${ }^{3}$, surge a possibilidade de se empregar essa tecnologia para estudar de forma incruenta outros aspectos da doença arterial coronária. Nesta edição da Revista Brasileira de Cardiologia Invasiva, Falcão et al. ${ }^{4}$ exploram uma dessas possibilidades, ao comparar a densidade radiológica da placa de ateroma pela angiotomografia às características da placa pelo ultrassom intracoronário com a técnica de histologia virtual.

Na verdade, a perspectiva de se analisar do ponto de vista tanto quantitativo como qualitativo a parede da artéria coronária e estimar a composição das placas obstrutivas tem despertado o interesse dos investigadores desde os primórdios da utilização desse método diagnóstico. Utilizando sistemas de quatro fileiras de detectores, Schroeder et al. ${ }^{5}$ avaliaram 15 pacientes pela tomografia e pelo ultrassom intracoronário, que serviu de padrão de referência, e classificaram as placas como moles, intermediárias e fibróticas. Os autores demonstraram grande concordância entre os exames, pois o coeficiente médio de atenuação de raios $X$ era significativamente diferente em cada um desses subtipos de placas ${ }^{5}$. Empregando metodologia de trabalho semelhante, mas com tomógrafos de 16 fileiras de detectores, Schoenhagen et al. ${ }^{6}$ chegaram a resultados semelhantes, mas destacaram o fato de que a concordância entre os dois exames de imagem para determinar a presença de remodelamento positivo era 0,90 .
Leber et al. ${ }^{7,8}$ também procuraram definir a composição das placas de ateroma, inicialmente com sistemas de 16 detectores $^{7}$ e posteriormente com sistemas de 64 detectores $^{8}$. A comparação dos resultados apresentada por esses autores permite concluir que ao utilizar os tomógrafos mais modernos os pesquisadores lograram, além de diferençar as placas em moles, intermediárias e fibróticas, identificar, em alguns ateromas, a presença de núcleos lipídicos e de pequenos pontos de calcificação em estenoses cujo maior componente era o de material não-calcificado. Schmid et al. ${ }^{9}$ destacaram o fato de que diferenças semelhantes a essas poderiam distinguir pacientes com síndromes coronárias agudas, pois estes apresentavam mais frequentemente baixo índice de atenuação radiológica e remodelamento positivo, características que poderiam apontar a existência de placas vulneráveis.

Por outro lado, Sun et al. ${ }^{10}$ chamaram a atenção para o fato de que, a despeito de a tomografia facultar a identificação e a quantificação do volume de aterosclerose que compromete a árvore arterial coronária, seu uso para a definição exata dos componentes da placa obstrutiva ainda não se encontra pronto para aplicação clínica, pelo menos nos sistemas de 64 detectores. Limitações também foram encontradas no estudo de Brodoefel et al. ${ }^{11}$, que, ao compararem os resultados da tomografia com os da histologia virtual, revelaram que a correlação entre a estimativa de composição dos ateromas pelos dois exames não era muito próxima, embora a definição dos componentes da placa pela tomografia seja altamente reprodutível. Choi et al. ${ }^{12}$ também compararam os resultados da tomografia aos da histologia virtual, destacando o fato de que o coeficiente de atenuação de placas não-calcificadas depende, em grande parte, do volume de necrose contido em cada uma das placas estudadas. Esses autores destacaram o fato de que, mesmo se as limitações atuais forem consideradas, esse tipo de achado pode vir a ter grande importância na prática clínica.

\footnotetext{
Instituto Dante Pazzanese de Cardiologia - São Paulo, SP, Brasil.

Correspondência: Ibraim Pinto. Av. Dr. Dante Pazzanese, 500 - Ibirapuera - São Paulo, SP, Brasil - CEP 04012-180

E-mail: ibraimp@uol.com.br

Recebido em: 9/9/2009 • Aceito em: 10/9/2009
} 
O estudo de Falcão et al. ${ }^{4}$ insere-se no mesmo contexto. Os próprios autores reconhecem que a correlação entre os componentes individuais da placa pelos dois exames é fraca, mas ressaltam que a tomografia se mostrou sensível à variação dos elementos presentes na parede arterial e no ateroma, conforme a aferição da histologia virtual, e que o volume de necrose presente na placa influencia a medida do coeficiente de atenuação radiológica pela tomografia.

Esses achados podem apresentar grande impacto na prática clínica. A tomografia das artérias coronárias foi introduzida inicialmente com o objetivo de quantificar o índice de calcificação nas artérias coronárias e, posteriormente, identificar ou excluir a presença de estenoses no leito arterial coronário. Por outro lado, não se pode esquecer que a tomografia faculta a visualização tanto da luz arterial como da parede vascular. Em outros territórios do corpo humano, como o crânio, a aorta e as vísceras abdominais, a interação dos raios $\mathrm{X}$ com os tecidos estudados possibilita identificar a presença de hematomas, gordura e fibrose. A extensão dessa abordagem para as paredes das artérias coronárias pareceu um fato natural e a relação dos trabalhos aqui discutidos confirma esse fato.

Contudo, não se pode esquecer que as artérias coronárias têm dimensões reduzidas, habitualmente inferiores a $3 \mathrm{~mm}$, e exibem movimentação complexa ao longo do ciclo cardíaco, consideradas limitações importantes para o estudo não-invasivo da parede arterial pela tomografia. Daí haver uma associação imperfeita entre os achados da tomografia e do ultrassom intracoronário ou da histologia virtual, conforme observado também no estudo de Falcão et al. ${ }^{4}$.

Mais que as limitações apresentadas nos estudos discutidos, deve-se destacar, porém, alguns aspectos positivos inferidos a partir dos dados apresentados nesse artigo. É claro que a qualidade das imagens tomográficas aumenta conforme avança a tecnologia, que se encontra sempre em evolução, sendo lícito esperar-se, portanto, que resultados ainda mais precisos venham a ser encontrados no futuro.

Mas o aspecto mais animador, a meu ver, é o fato de que todos os autores que se dedicaram a esse tipo de pesquisa, tais como Falcão et al. ${ }^{4}$, lograram demonstrar que a tomografia fornece imagens que refletem as alterações anatômicas e estruturais decorrentes da evolução da aterosclerose nas artérias coronárias. Esse exame apresenta-se, assim, como a única metodologia não-invasiva que, no momento, possibilita a monitorização não-invasiva da doença arterial coronária. A elaboração de trabalhos como o aqui discutido pode levar ao desenvolvimento de um instrumental que possibilite aos pesquisadores obter informações dinâmicas e confiáveis sobre a aterosclerose coronária de modo não-invasivo.

Resta aos que trabalham com exames de diagnóstico por imagem vencer esses novos obstáculos e desvendar os horizontes para oferecer à comunidade cardiológica mais um importante avanço.

\section{CONFLITO DE INTERESSES}

O autor declarou inexistência de conflito de interesses.

\section{REFERÊNCIAS BIBLIOGRÁFICAS}

1. Stein PD, Yaekoub AY, Matta F, Sostman HD. 64-slice CT for diagnosis of coronary artery disease: a systematic review. Am J Med. 2008;121(8):715-25.

2. Gopalakrishnan P, Wilson GT, Tak T. Accuracy of multislice computed tomography coronary angiography: a pooled estimate. Cardiol Rev. 2008;16(4):189-96.

3. Weustink AC, Nieman K, Pugliese F, Mollet NR, Meijboom BW, van Mieghem C, et al. Diagnostic accuracy of computed tomography angiography in patients after bypass grafting: comparison with invasive coronary angiography. JACC Cardiovasc Imaging. 2009;2(7):816-24.

4. Falcão JLAA, Gurudevan SV, Shiozaki AA, Coelho Filho OR, Falcão BAA, Sales FJR, et al. Associação entre a densidade radiológica da placa à tomografia de coronárias com 64 colunas de detectores e a composição da placa ao ultrassom intravascular com técnica de histologia virtual: resultados de uma comparação pareada prospectiva. Rev Bras Cardiol Invas. 2009;17(3):327-34.

5. Schroeder S, Kopp AF, Baumbach A, Meisner C, Kuettner A, Georg C, et al. Noninvasive detection and evaluation of atherosclerotic coronary plaques with multislice computed tomography. J Am Coll Cardiol. 2001;37(5):1430-5.

6. Schoenhagen P, Tuzcu EM, Stillman AE, Moliterno DJ, Halliburton SS, Kuzmiak SA, et al. Non-invasive assessment of plaque morphology and remodeling in mildly stenotic coronary segments: comparison of 16-slice computed tomography and intravascular ultrasound. Coron Artery Dis. 2003; 14(6):459-62.

7. Leber AW, Knez A, Becker A, Becker C, von Ziegler F, Nikolaou $\mathrm{K}$, et al. Accuracy of multidetector spiral computed tomography in identifying and differentiating the composition of coronary atherosclerotic plaques: a comparative study with intracoronary ultrasound. J Am Coll Cardiol. 2004;43(7):1241-7.

8. Leber AW, Becker A, Knez A, von Ziegler F, Sirol F, Nikolaou $K$, et al. Accuracy of 64-slice computed tomography to classify and quantify plaque volumes in the proximal coronary system: a comparative study using intravascular ultrasound. J Am Coll Cardiol. 2006;47(3):672-7.

9. Schmid M, Pflederer T, Jang IK, Ropers D, Sei K, Daniel WG, et al. Relationship between degree of remodeling and CT attenuation of plaque in coronary atherosclerotic lesions: an in-vivo analysis by multi-detector computed tomography. Atherosclerosis. 2008;197(1):457-64.

10. Sun J, Zhang A, Lu B, Yu W, Yang Y, Zhou Y, et al. Identification and quantification of coronary atherosclerotic plaques: a comparison of 64-MDCT and intravascular ultrasound. AJR Am J Roentgenol. 2008;190(3):748-54.

11. Brodoefel H, Reimann A, Heuschmid M, Tsiflikas I, Kopp AF, Schroeder S, et al. Characterization of coronary atherosclerosis by dual-source computed tomography and HU-based color mapping: a pilot study. Eur Radiol. 2008;18(11):2466-74.

12. Choi BJ, Kang DK, Tahk SJ, Choi SY, Yoon MH, Lim HS, et al. Comparison of 64-slice multidetector computed tomography with spectral analysis of intravascular ultrasound backscatter signals for characterizations of noncalcified coronary arterial plaques. Am J Cardiol. 2008;102(8):988-93. 